\title{
Padrões de Interação Genitores-Crianças com e sem Síndrome de Down
}

\author{
Patterns of Interaction Between Parents and their Children \\ with and without Down Syndrome
}

\author{
Nara Liana Pereira Silva* ${ }^{*}, \&$ Maria Auxiliadora Dessen ${ }^{b}$ \\ Instituto de Educação Superior de Brasília, Brasília, Brasil ${ }^{a}$; Universidade de Brasília, Brasília, Brasil ${ }^{b}$
}

\begin{abstract}
Resumo
A literatura a respeito das interações familiares de crianças com síndrome de Down é escassa, sobretudo em relação à participação do pai. Este estudo compara as interações familiares entre dois grupos de crianças préescolares, um com e outro sem síndrome de Down, enfatizando as continuidades e mudanças nos seus estilos de interação, ao longo de dois anos. Dez famílias foram visitadas semestralmente em suas casas e as interações entre criança-mãe, criança-pai e criança-mãe-pai ocorridas durante 'atividades livres' foram gravadas em vídeo. Foram analisados 1050 minutos de gravação por meio de observação direta. Os resultados mostram que há similaridades, mas também diferenças na frequiência de atividades realizadas e na participação e qualidade das interações entre genitores-criança e que as famílias diferem quanto aos padrões de estabilidades e mudanças, no decorrer do tempo. Os dados sugerem que há necessidade de incluir o pai e os irmãos nos planejamentos de pesquisa e analisar os dados observacionais considerando díades, tríades e tétrades.

Palavras-chave: Interações familiares; relações parentais; crianças com síndrome de Down; metodologia de observação direta.
\end{abstract}

\begin{abstract}
Literature about family interactions of Down's syndrome children is scarce, especially regarding the father's involvement. This study compares the family interactions between two groups of pre-school children, one with Down's syndrome and the other with a typical development, highlighting the continuities and changes in their styles of family interaction for a period of two years. Ten families were visited in their homes and the child-mother, child-father, and child-mother-father interactions that developed during 'free activities' were recorded on video. 1050 minutes of video were analyzed by direct observation. The results show us that there are similarities but also differences in the frequency of activities, family member participation, and quality of parent-child interactions. And, that the families are dissimilar as to the patterns of stability and change, as time elapses. We suggest that it is very important to include the father and siblings in researches and to analyze the observational data considering the interactions of dyads, triads, and tetrads.

Keyzords: Family interactions; parental relationships; Down syndrome children; direct observation methodology.
\end{abstract}

Os estudos na área de deficiência mental e família, especialmente aqueles que se referem às investigações sobre as relações parentais, têm focalizado dois aspectos primordiais para a compreensão do desenvolvimento: (a) como e de que forma os genitores comportam-se e respondem às suas crianças e (b) como os aspectos do comportamento parental afetam e são afetados pelos diferentes aspectos do comportamento e desenvolvimento da criança (Berger, 1990). Para responder às questões de pesquisa, os pesquisadores têm recorrido cada vez mais à abordagem sistêmica da família, que prioriza não só a unidade mínima de análise, isto é, as díades, como também os demais níveis, as

\footnotetext{
${ }^{*}$ Endereço para correspondência: Universidade de Brasília - UnB, Instituto de Psicologia/PED, Laboratório de Desenvolvimento Familiar, Campus Universitário Darcy Ribeiro, Brasília, DF, 709 10-90o Fone: (061) 3307 2625, Ramais: 409 ou 417; Fax: (061)3347 7746. E-mail: liana@unb.br Este artigo é baseado em parte dos dados da tese de Doutorado apresentada ao Instituto de Psicologia da Universidade de Brasília pela primeira autora, sob a orientação da segunda. As autoras agradecem o apoio concedido pelo CNPq.
}

tríades e tétrades, levando em consideração as inter-relações do sistema familiar com os demais sistemas ecológicos (Dessen, 1997; Kreppner 2000). A adoção do modelo sistêmico tem contribuído para a produção de um conhecimento relevante sobre as relações familiares e para unificar as pesquisas na área da psicologia da família.

Para compreender melhor a dinâmica do funcionamento das famílias, é necessário focalizar todos os subsistemas familiares, incluindo, além dos subsistemas parental e conjugal, o de irmão-irmão, avós-netos, dentre outros considerados relevantes para responder as questões do estudo. Nesta pesquisa, priorizamos o subsistema genitores-criança, pois as interações desenvolvidas neste subsistema exercem influência preponderante no desenvolvimento da criança, com implicações para os estágios subseqüentes do curso de vida (Kreppner, 2000). Mas, as análises do subsistema parental devem considerar não apenas as interações diádicas pai-criança e mãe-criança, mas também as tríades, pois estas apresentam características específicas que não são vistas, por exemplo, na interação triádica 
pai-mãe-criança (Dessen, 1997; Kreppner, 2000). É interessante ressaltar que as pesquisas realizadas com tríades, na área de deficiência mental, ainda são escassas, tanto no exterior quanto no Brasil; com menos de $10 \%$ das pesquisas estrangeiras focalizando o subsistema pai-mãe-criança (Dessen \& Pereira-Silva, 2000).

O subsistema mãe-criança é o que mais tem sido investigado na literatura (Cielinski, Vaughn, Seifer \& Contreras, 1995; Colnago, 1991; Landry, Garner, Pirie \& Swank, 1994; Lieberman, Padan-Belkin \& Harel, 1995; Marfo, Dedrick \& Barbour, 1998; Roach, Barratt, Miller \& Leavitt, 1998; Sigolo, 1994, 2004; Vaughn et al., 1994). De acordo com esses autores, apesar da existência de algumas divergências nos resultados, há dados consistentes no que tange, principalmente, a uma maior diretividade das mães de crianças com deficiência mental em relação às mães de crianças com desenvolvimento típico, quando elas interagem com suas crianças.

A esmagadora consistência nos resultados de pesquisas sobre diretividade e a escassez de estudos focalizando as variações intra-grupos têm ajudado a perpetuar a visão de que as mães de crianças com deficiência mental compartilham uma tendência generalizada para dirigir o comportamento de seus filhos. Embora haja pouca evidência de que a diretividade materna tenha uma influência negativa sobre a adaptação e responsividade da criança com síndrome de Down (Cielinski et al., 1995; Landry et al., 1994; Roach et al., 1998), esta questão precisa ser tratada de uma forma mais complexa. Primeiro, porque a conotação negativa da diretividade está, em parte, associada a uma linha de pesquisa do desenvolvimento da linguagem, datando da década de 70 , em que os efeitos potencialmente negativos do controle maternal e da diretividade eram muito ressaltados. Uma outra visão que tem contribuído para essa idéia negativa da diretividade é a de inferir ou associar a deficiência à 'diferença'.

Marfo et al. (1998), na tentativa de diminuir a prevalência de uma visão negativa em relação à diretividade das mães, chamam a atenção para o fato de que é importante analisar a diretividade como uma característica do estilo interacional materno de mães de crianças com deficiência mental, reconhecendo a necessidade adaptativa do comportamento parental ao comportamento da criança. Além deste aspecto, há muitos outros que caracterizam o estilo de interação dessas mães que deveriam ser evidenciados nas pesquisas. Nesta direção, Roach et al. (1998) realizaram um estudo com 28 crianças com síndrome de Down e 56 com desenvolvimento típico, das quais 28 tinham a mesma idade cronológica e 28 a mesma idade de desenvolvimento das crianças com síndrome de Down. Os resultados indicam que as mães de crianças com síndrome de Down apresentam uma freqüência significativamente alta de comportamentos de apoio comparadas às mães de crianças com desenvolvimento típico. Elas também elogiam mais freqüentemente as suas crianças que as outras mães, embora todas elas tenham respondido seletivamente com elogio, em um esforço para reforçar as tentativas precoces de suas crianças em uma situação estruturada de jogo com objetos. Além disso, nessa situação, não houve qualquer diferença entre crianças com síndrome de Down e com desenvolvimento típico.

Alguns estudos mostram também que as interações diádicas mãe-criança são iniciadas mais freqüentemente pelas mães que pelas crianças com deficiência mental (Landry et al., 1994; Pereira-Silva \& Dessen, 2003; Sigolo, 1994); no entanto à medida que o bebê com síndrome de Down cresce, este passa a tomar a iniciativa na realização de atividades, especialmente no que tange ao banho e à alimentação (Colnago, 1991). Outras características das interações têm sido ressaltadas recentemente; por exemplo, Pereira-Silva e Dessen (2003), ao analisarem a qualidade das interações em seis famílias de crianças com síndrome de Down, verificaram que as interações mãecriança eram mais amistosas (96\%) que conflituosas, mais sincrônicas $(76,7 \%)$ que sem sincronia.

Apesar dos esforços, a ênfase dos estudos ainda continua sendo sobre a díade mãe-criança com deficiência, em detrimento das interações diádicas pai-criança (Dessen \& Pereira-Silva, 2000), a despeito de a literatura ressaltar a necessidade da inclusão do pai nos planejamentos de pesquisa (Dessen \& Lewis, 1998). Alguns estudos indicam que os pais de crianças com deficiência mental apresentam comportamentos de comunicação significativamente menos positivos e altas taxas de reciprocidade negativa em comparação com pais de crianças com desenvolvimento típico e com mães de crianças com deficiência mental (Floyd, Costigan \& Phillippe, 1997; Floyd \& Zmich, 1991). Já, os resultados do estudo de Pereira-Silva e Dessen (2003), realizado no Brasil, apontam na direção de pais participativos e com uma freqüência de iniciação de interações mais elevada que as mães, sugerindo possíveis diferenças culturais que precisam ser investigadas mais profundamente.

Rodrigue, Morgan e Geffken (1992), estudando a adaptação psicossocial de pais de crianças autistas, com síndrome de Down e com desenvolvimento típico, não encontraram diferenças significativas entre os três grupos quanto aos cuidados com os filhos e à satisfação percebida pelos mesmos. Mas, os pais de meninos relataram maior satisfação com a paternidade que os pais de meninas. No entanto, os resultados do estudo de Hornby (1995) não mostram contrastes entre a adaptação dos pais às filhas e aos filhos com síndrome de Down. Neste estudo, a adaptação do pai estava relacionada à satisfação com o apoio social recebido, à satisfação marital e às características de personalidade, mais do que às características de sua criança deficiente. A severidade da deficiência é outra variável que tem influência no envolvimento do pai; contudo, a literatura mostra dados contraditórios também a esse respeito, com algumas pesquisas apontando para um maior envolvimento, enquanto outras não mostram diferenças (Hornby, 1995; Lamb \& Billings, 1997).

Portanto, os dados atuais refletem a inconsistência existente na área, sugerindo a necessidade de mais pesquisas que incluam não somente o pai em seus planejamentos, 
mas também outros componentes da família, particularmente os irmãos. Assim, considerando a inconsistência dos dados, a escassez de pesquisas sobre as interações familiares de crianças com síndrome de Down em nosso contexto cultural e a necessidade de compreender mais profundamente os padrões de comunicação visando o planejamento de programas de educação familiar, implementamos um estudo com o objetivo principal de descrever algumas das dimensões das relações parentais nessas famílias. Dentre as dimensões investigadas, destacamos os padrões de interação familiar referentes ao conteúdo (atividades realizadas), estrutura de participação dos membros familiares nas atividades, modos de transição de uma atividade para outra e qualidade das interações genitores-criança. Também constituem objetivos desse estudo identificar aspectos similares e diferenciadores do funcionamento das famílias de crianças com síndrome de Down em relação a famílias de crianças com desenvolvimento típico e descrever os padrões de continuidades e mudanças na comunicação familiar, no decorrer de dois anos, para ambos os tipos de famílias.

\section{Método}

\section{Participantes}

Participaram deste estudo dez famílias compostas por mãe, pai e criança-alvo, sendo cinco com síndrome de Down (SD) e cinco com desenvolvimento típico (DT) ${ }^{1}$. Todos os membros da família moravam juntos em cidades satélites de Brasília $(n=9)$ ou no Plano Piloto $(n=1)$, sendo quatro crianças do sexo masculino e seis do sexo feminino, com idades variando entre dois a quatro anos, por ocasião da coleta de dados.

Nas famílias de crianças SD, a idade média das mães era de 37 anos e dos pais 33; e, nas famílias de crianças DT, de 33 e 35 anos, respectivamente. Os pais das crianças SD exerciam as seguintes ocupações: radialista, administrador (aposentado), autônomo, militar e ajudante de serviços gerais; e os pais das crianças DT: vendedor, funcionário público, militar e auxiliar de escritório. Enquanto a maioria das mães das crianças $\mathrm{SD}(n=3)$ trabalhava fora de casa, a de crianças DT $(n=3)$ não trabalhava fora de casa. A renda familiar média era de 7,2 salários mínimos².

\section{Procedimentos}

A coleta de dados com as dez famílias foi efetuada somente pela pesquisadora, na própria residência das mesmas. As famílias foram visitadas a cada seis meses, perfazendo um total de quatro etapas de coleta de dados com as famílias das crianças SD e de três etapas com as famílias das crianças DT. Foram realizadas 60 sessões de observação com as

\footnotetext{
${ }^{1}$ Crianças com desenvolvimento típico referem-se àquelas que não apresentam qualquer tipo de deficiência, de acordo com os relatos dos genitores e de suas professoras.

${ }^{2}$ A renda familiar foi calculada com base no salário mínimo de $\mathrm{R} \$ 136,00$ para as famílias de crianças SD e $\mathrm{R} \$ 151,00$ para as famílias de crianças DT, de acordo com a data de início da coleta de dados.
}

famílias das crianças SD e 45 sessões com as famílias das crianças DT, totalizando 1050 minutos de gravação em vídeo, em situações diádicas, com a presença da criança e a da mãe (C-M) e da criança e do pai (C-P), e triádicas, envolvendo a criança, a mãe e o pai (C-M-P). A instrução dada às famílias era para que 'fizessem o que estavam acostumadas a fazer durante o seu tempo livre em casa'.

Os dados observacionais foram analisados de acordo com o modelo proposto por Dessen (1992), tendo o sistema de categorias para análise de interações familiares sido adaptado para o estudo em questão ${ }^{3}$. A concordância entre-observadores foi calculada com base em 16,6\% das sessões $(n=9)$ referentes à primeira fase de coleta de dados com as famílias de crianças SD, tendo sido obtidos os seguintes índices: (a) atividades realizadas: $75 \%$; (b) estrutura de participação: 64,5\%; (c) transições: 60\%; (d) qualidade das interações: 63\%; (e) categorias comportamentais: $79 \%$. O índice global de concordância foi de $68,7 \%$. A concordância intra-observador foi calculada com base em $10 \%$ do total das sessões de observação, escolhidas de acordo com os tipos de situação. Os índices obtidos foram: (a) atividades realizadas: 56,3\%; (b) estrutura de participação: 62,7\%; (c) início de interações: 56\%; (d) términos: 43\%; (e) transições: $63 \%$; (f) qualidade das interações: 53,3\%; e (g) categorias comportamentais: 63,3\%. O índice global de concordância foi de 54,4\%.

\section{Resultados}

\section{Padrões de Comunicação Familiar}

\section{Conteúdo das interações parentais.}

O que os genitores e sua criança fazem juntos? As atividades desenvolvidas pelas famílias foram diversificadas; porém, houve predominância das atividades 'Lúdicas' em detrimento das demais. As atividades realizadas mais freqüentemente pelas díades (C-M e C-P) foram as 'Brincadeiras' (42,5\%), seguidas por 'Conversar' (20\%), 'Assistir à televisão' (10,9\%), 'Socializar' (9,5\%) e 'Atividades artísticas' (8,6\%). O tipo de brincadeira mais freqüente foi a 'Brincadeira com objetos' (21,6\%), enquanto a atividade de 'Conversar' girou sobre o tema 'estímulos presentes' $(16,7 \%)$. Houve similaridade nos tipos de atividades desenvolvidas por ambos os tipos de famílias. Contudo, os 'Jogos' e as 'Brincadeiras de atividades escolares' foram mais freqüentes nas famílias DT (C-M: 2\% e C-P: 4,1\%; C-M: $16,1 \%$ e C-P: $8,2 \%$, respectivamente) que nas famílias SD (C-M: O\% e C-P: 0,3\%; C-M: 3,3\% e C-P: $2 \%$, respectivamente). Por outro lado, 'Socializar' e 'Contar histórias' foram mais freqüentemente desenvolvidas nas famílias SD (C-M: 10,8\% e C-P: 12,1\%; C-M: 9,4\% e C-P: $2,6 \%$, respectivamente) que nas DT (C-M: $10,1 \%$ e C-P: $2,5 \%$; C-M: O,7\% e C-P: 1,7\%, respectivamente).

Quando ambos os genitores participavam das atividades com seus filhos (C-M-P), as 'Brincadeiras' (35,5\%)
${ }^{3}$ Para a definição das categorias, consultar Pereira-Silva (2003). 
continuaram sendo as atividades predominantes, seguidas por 'Conversar' (30,3\%) e 'Socializar' (11\%). Eles conversaram mais freqüentemente 'Sobre estímulos presentes' (23\%) e 'Brincaram com objetos'(15,4\%). Com relação às diferenças entre os tipos de família, no que tange ao engajamento triádico, os 'Jogos' (3,5\%) ocorreram apenas nas famílias DT, enquanto as 'Brincadeiras de manipular o corpo' (14\%), 'Socializar' (13,8\%) e as 'Atividades artísticas' (9,2\%) ocorreram com mais freqüência nas famílias SD. 'Conversar' e 'Contar histórias' foram mais freqüentes nas famílias DT (36,3\% e 9,9\%, respectivamente) que nas famílias SD (25,8\% e $1 \%$, respectivamente), denotando um padrão diferente daquele ocorrido nas díades.

Estrutura de participação familiar e transições de atividades Como os genitores e sua criança participam das atividades? A participação 'Conjunta' foi a mais freqüente (85,5\%), seguida pela 'Paralela' (9,2\%) e, por último, pela 'Individual' (5,2\%). Não houve diferença entre as famílias no que se refere ao tipo de participação mais freqüente, tanto em díades quanto em tríades. O tipo de participação 'Paralela' foi mais freqüente quando a criança estava junto com a mãe e o pai $(11,3 \%)$ em famílias SD, enquanto nas famílias DT, este tipo de participação ocorreu com maior freqüência na situação que envolvia apenas a criança e o pai (C-P = 14,7\%). É interessante destacar que as mães, nas famílias DT, quando sozinhas com sua criança, engajaram-se em atividades predominantemente de forma 'Conjunta' (92,8\%).

De que modo os genitores e sua criança mudam de uma atividade para outra? As mudanças ocorreram mais freqüentemente de forma 'Direta' $(78,1 \%)$ que pela 'Dissolução do Grupo' (13,2\%) ou pela 'Negociação' (8,7\%). Não houve diferenças entre os dois tipos de família no que se refere aos estilos de transição. Tanto nas situações diádicas quanto nas triádicas, a transição 'Direta' foi mais freqüente, permanecendo este padrão nos dois grupos de família. A 'Dissolução do grupo' foi menos freqüente na situação C-M (7,7\%) que nas demais situações, nas famílias DT. A situação C-M-P favoreceu um aumento da 'Dissolução do grupo' em ambos os tipos de família (SD = $17,5 \%, \mathrm{DT}=12 \%$ ) em comparação com as situações diádicas C-M e C-P. Já, as 'Negociações' foram mais freqüentes na situação C-P (SD = 11,9\%; DT = 10,6), apesar de haver um percentual similar na situação C-M (SD = $9,2 ; \mathrm{DT}=10 \%)$. Isto significa que pais e mães, quando sozinhos com sua criança, utilizam modos similares para mudarem de atividade.

\section{Qualidade das interações parentais.}

Qual a qualidade das interações entre os genitores e sua criança? As interações entre a criança e seus genitores caracterizaram-se pela 'Sincronia' (91,8\%), 'Supervisão' (98\%), 'Amistosidade' (98,9\%) e 'Liderança' (94,9\%). A Tabela 1 apresenta as freqüências relativas e os percentuais das categorias referentes aos aspectos de 'Sincronicidade', 'Supervisão', 'Amistosidade' e 'Liderança' das interações desenvolvidas nos subsistemas C-M, C-P e C-M-P, de acordo com os tipos de família.

\section{Tabela 1}

Freqüências Relativas e Percentuais das Dimensões de Qualidade das Interações, com Especificação dos Subsistemas Familiares

\begin{tabular}{|c|c|c|c|c|c|c|c|c|c|c|c|c|c|c|}
\hline \multirow{4}{*}{ 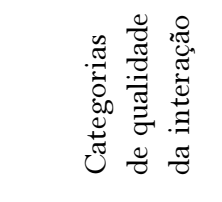 } & \multicolumn{12}{|c|}{ Subsistemas familiares } & \multirow{3}{*}{\multicolumn{2}{|c|}{ Total }} \\
\hline & \multicolumn{6}{|c|}{ Síndrome de Down } & \multicolumn{6}{|c|}{ Desenvolvimento típico } & & \\
\hline & $\mathrm{C}-\mathrm{M}$ & & C-P & & C-M-P & & $\mathrm{C}-\mathrm{M}$ & & C-P & & $\mathrm{C}-\mathrm{M}-\mathrm{I}$ & & & \\
\hline & Freq. & $\%$ & Freq. & $\%$ & Freq. & $\%$ & Freq. & $\%$ & Freq. & $\%$ & Freq. & $\%$ & Freq. & $\%$ \\
\hline Com sincronia & 101,25 & 80,2 & 106,5 & 81,3 & 123 & 83,2 & 346,7 & 98,0 & 272,3 & 97,3 & 279 & 97,7 & 1228,75 & 92,8 \\
\hline Sem sincronia & 25 & 19,8 & 24,5 & 18,7 & 24,75 & 16,8 & 6,7 & $2, \mathrm{O}$ & 7,7 & 2,7 & 6,7 & 2,3 & 95,35 & 7,2 \\
\hline Com supervisão & 122,5 & 97,0 & 129,75 & 99,0 & 142,75 & 96,6 & 347 & 98,2 & 272,7 & 97,4 & 281,7 & 98,6 & 1296,4 & 98,0 \\
\hline Sem supervisão & 3,75 & 3,0 & 1,25 & $1, \mathrm{O}$ & 5 & 3,4 & 6,4 & 1,8 & 7,3 & 2,6 & 4 & 1,4 & 27,7 & 2,0 \\
\hline Amistosa & 125 & 99,0 & 124,75 & 95,2 & 143,75 & 97,3 & 352,7 & 99,8 & 280 & 100 & 285,7 & 100 & 1311,9 & 99,0 \\
\hline Conflituosa & 1,25 & $1, \mathrm{O}$ & 6,25 & 4,8 & 4 & 2,7 & 0,7 & 0,2 & - & 一 & - & 一 & 12,2 & 1,0 \\
\hline Com liderança & 118,25 & 93,7 & 122,5 & 93,5 & 142 & 96,0 & 334,7 & 94,7 & 261,3 & 93,3 & 278 & 97,3 & 1256,75 & 94,9 \\
\hline Sem liderança & 8 & 6,3 & 8,5 & 6,5 & 5,75 & 4,0 & 18,7 & 5,3 & 18,7 & 6,7 & 7,7 & 2,7 & 67,35 & 5,1 \\
\hline
\end{tabular}

Nota. Cada dimensão totaliza $100 \%$, uma vez que suas categorias são mutuamente exclusivas.

Quem 'supervisiona' e 'lidera' as interações familiares? As mães, em ambos os tipos de família, são as maiores responsáveis pela 'Supervisão' das interações familiares $(\mathrm{SD}=42,4 \%, \mathrm{DT}=55,6 \%)$, seguidas pelos pais $(\mathrm{SD}=$
$41 \%$, DT $=38,1 \%$ ). A criança, nas famílias SD, foi responsável por 'Supervisionar' em apenas $0,8 \%$ dos episódios interativos enquanto a criança, nas famílias DT, foi responsável por 'Supervisionar' 1,6\% dos episódios. 
Quanto à 'Liderança', esta foi exercida mais freqüentemente pela mãe $(35,6 \%)$ e pelo pai $(34 \%)$, nas famílias SD, sendo a criança responsável por $17,5 \%$ da 'Liderança' exercida nas interações. Já nas famílias DT, a mãe 'Liderou' 36,8\% dos episódios, enquanto o pai 'Liderou' 25,9\% e a criança $30 \%$. Portanto, nas famílias DT, as crianças exerceram mais 'Liderança' que o pai e, também, que as crianças SD.

Estabilidades e Mudanças nos Padrões de Comunicação Parental

O que muda e o que permanece estável nas relações parentais, ao longo do tempo?

Há mudanças nas atividades realizadas? As famílias SD mostram uma tendência crescente de engajamento em 'Brincadeiras', enquanto as famílias DT mostram um padrão mais descontínuo no desenvolvimento deste tipo de atividade, cuja freqüência foi mais elevada na segunda fase de coleta de dados. As 'Brincadeiras Com objetos' decrescem na quarta fase, nas famílias SD, enquanto nas famílias DT, este tipo de brincadeira segue a tendência geral apresentada por essas famílias no que tange às brincadeiras. A Figura 1 apresenta as freqüências absolutas da atividade de 'Brincadeira', com destaque para a 'Brincadeira com objetos' (Figura 2), realizadas pelos genitores e sua criança.

\section{$\square$ Famílias SD $\square$ Famílias DT}
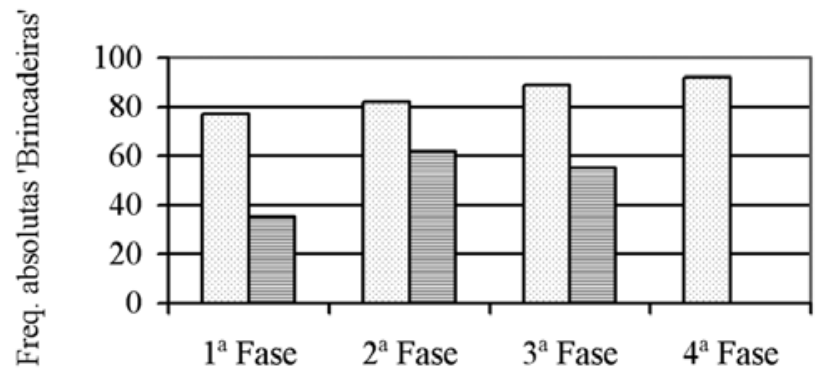

Figura 1. 'Brincadeiras' entre genitores-criança, no decorrer do tempo.

\section{$\square$ Famílias SD $\square$ Famílias DT}

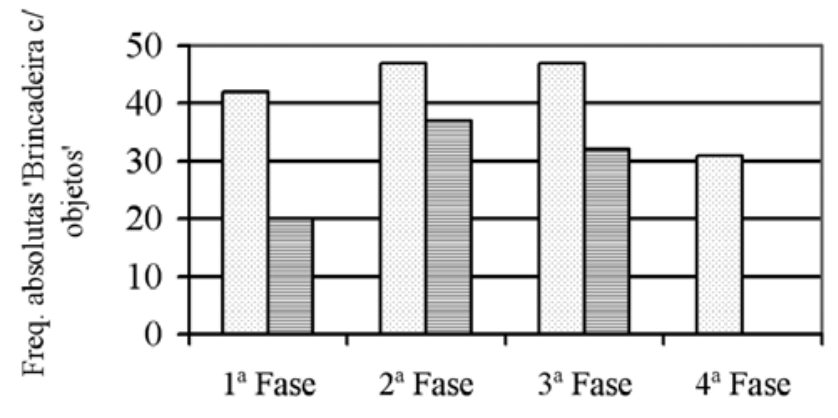

Figura 2. Brincadeira com objetos' durante as interações genitores-criança, no decorrer do tempo.

Há alteração na estrutura de participação? Ao longo do tempo, verifica-se que os genitores e sua criança, em am- bos os tipos de família, mantêm constante o padrão de participação 'Conjunto' nas atividades, embora nas famílias DT tenha ocorrido um ligeiro aumento de sua freqüência na $2^{\text {a }}$ fase e um declínio na $3^{\text {a }}$ fase. Já a participação 'Paralela' caracteriza-se por um padrão descontínuo (ora crescente, ora decrescente), particularmente nas famílias SD. A estrutura de participação 'Individual' apresenta, também, um padrão descontínuo, mas somente nas famílias SD, enquanto nas DT observa-se um padrão decrescente, ao longo do tempo. Esses padrões de continuidades e mudanças podem ser visualizados nas Figuras 3, 4 e 5.

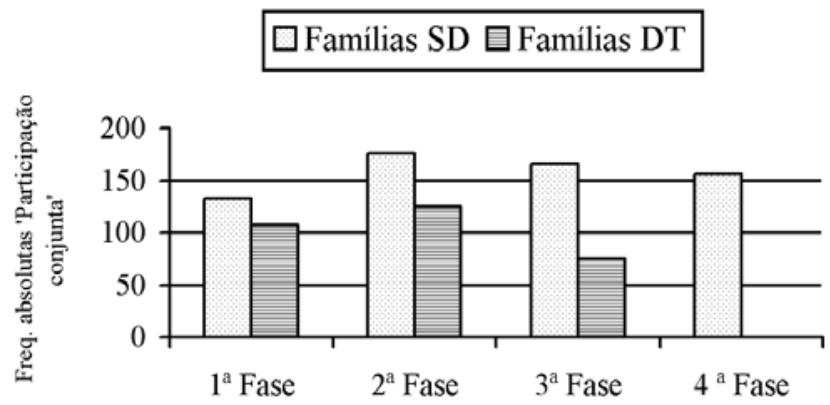

Figura 3. Participação 'Conjunta', ao longo do tempo.

口Famílias SD $\square$ Famílias DT

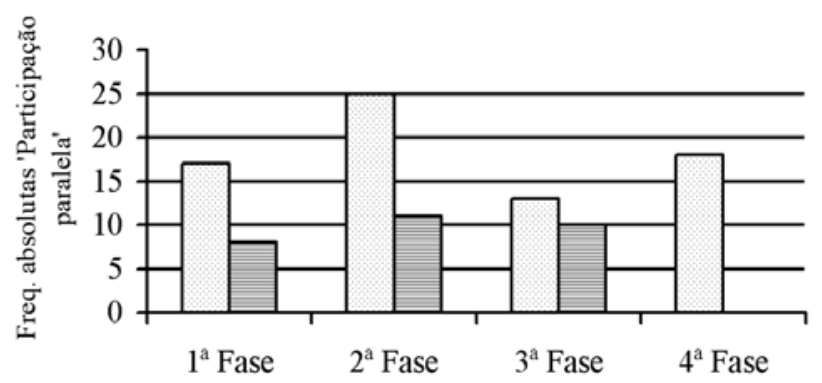

Figura 4. Participação 'Paralela', ao longo do tempo. $\square$ Famílias SD $\square$ Famílias DT

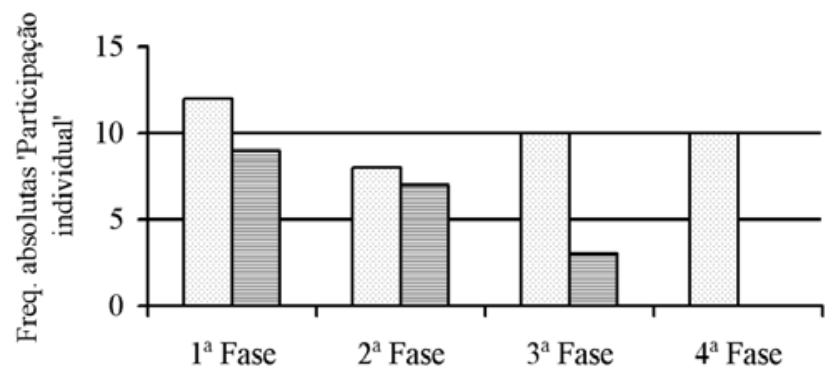

Figura 5. Participação 'Individual', ao longo do tempo.

Os padrões de transição familiar mudam ao longo do tempo? A análise das transições nas situações C-M, C-P e C-M-P revela que, na $2^{\text {a }}$ fase, tanto as famílias SD quanto 
as DT apresentam freqüências mais elevadas de transições 'Direta', havendo um ligeiro declínio na $3^{\text {a }}$ fase. Mas, as famílias DT mantêm uma estabilidade nas transições pela 'Dissolução do grupo', ao longo do tempo, enquanto as famílias SD apresentam um aumento e um decréscimo neste tipo de transição. É interessante destacar que as 'Negociações' tendem a aumentar nas famílias SD e a decrescer nas famílias DT, com o passar do tempo. As Figuras 6, 7 e 8 apresentam os padrões de continuidades e mudanças nas transições de uma atividade para outra.



Figura 6. Transição 'Direta', ao longo do tempo.

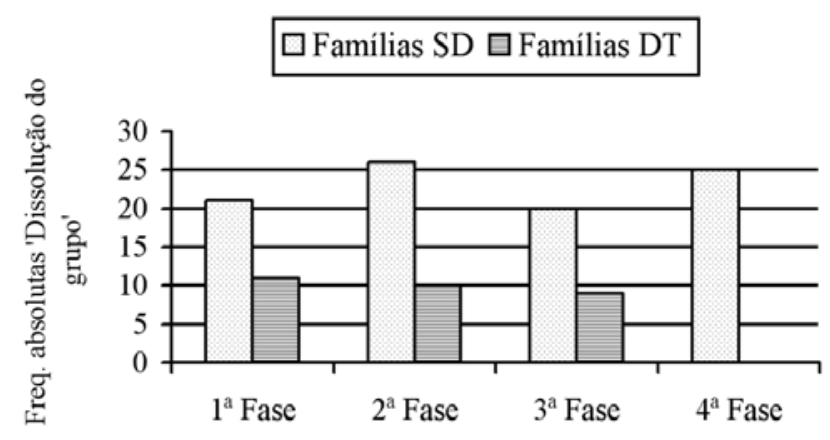

Figura 7. Transição pela 'Dissolução do grupo', ao longo do tempo.

\section{$\square$ Famílias SD $⿴$ GFamílias DT}

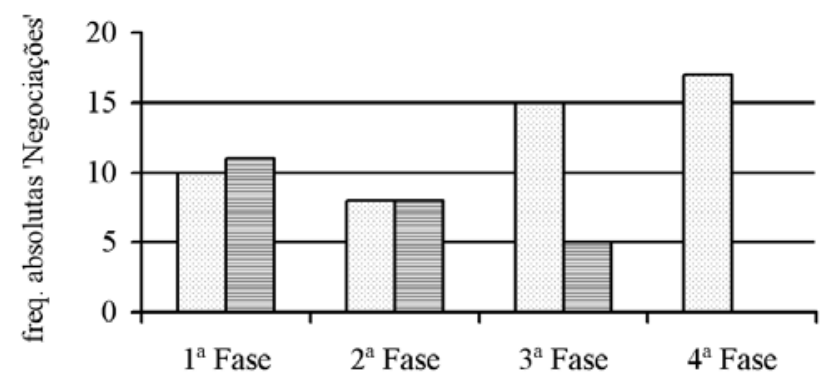

Figura 8. Transição por 'Negociação', ao longo do tempo.

A qualidade das interações genitores-criança é alterada no decorrer do tempo?_Destacamos aqui apenas as dimensões de 'Sincronia' e 'Amistosidade'. A Figura 9 mostra que as interações 'Sincrônicas' entre os genitores e sua criança SD permanecem estáveis ao longo do tempo, en- quanto nas famílias DT há uma tendência crescente deste padrão de interação. Nesse mesmo período, ambos os tipos de família apresentaram uma tendência à diminuição de episódios 'Sem sincronia' (Figura 10).

\section{$\square$ Familias SD $\square$ Famílias DT}

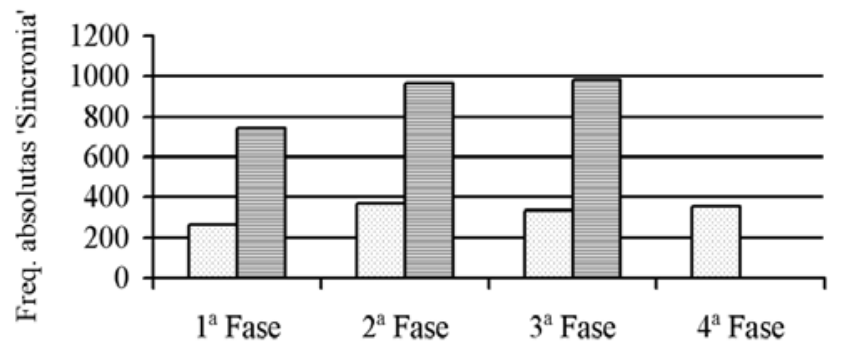

Figura 9. Interações 'Sincrônicas' entre genitores-criança, no decorrer do tempo.

\section{囲Famílias SD 曰Famílias DT}

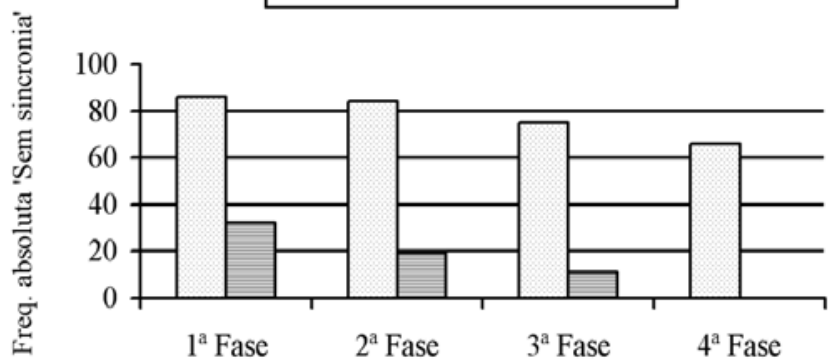

Figura 10. Interações 'Sem sincronia' entre genitores-criança, no decorrer do tempo.

Com relação à 'Amistosidade' nas interações das famílias, observamos que as famílias SD mantêm uma estabilidade na freqüência desse padrão, enquanto as famílias DT mostram uma tendência em aumentar os episódios de interação 'Amistosos'. Embora mantendo estabilidade no que se refere aos episódios 'Amistosos', as famílias SD diminuíram a freqüência de interações 'Conflituosas', ao longo do tempo. As Figuras 11 e 12 apresentam esses padrões de continuidades e mudanças da qualidade das interações parentais.

\section{Famílias SD $\square$ Famílias DT}

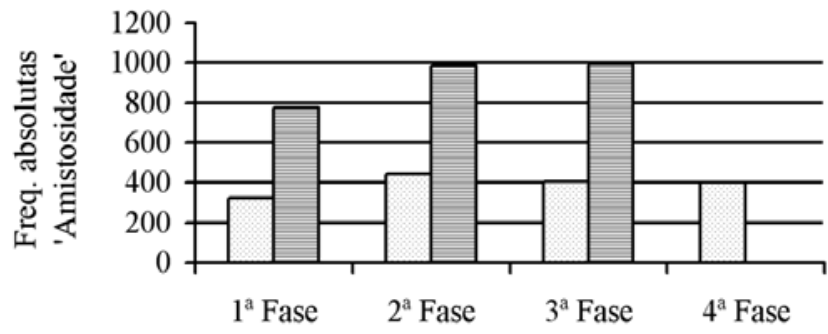

Figura 11. Interações 'Amistosas' entre genitores-criança, ao longo do tempo. 


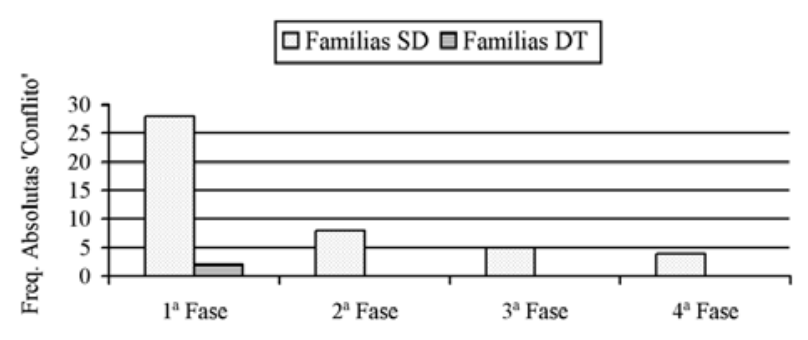

Figura 12. Interações 'Conflituosas' entre genitores-criança, ao longo do tempo.

\section{Discussão}

Focalizar a família enquanto grupo e, conseqüentemente, as influências e as inter-relações entre os diversos subsistemas familiares tornam a visão do desenvolvimento da família e dos indivíduos mais completa. No entanto, é preciso, também, incluir outros ambientes de desenvolvimento em que a criança está inserida, além da família, para que a nossa compreensão seja ainda mais completa. Neste particular, a adoção da perspectiva bioecológica de Bronfenbrenner (Bronfenbrenner, 1999; Bronfenbrenner \& Morris, 1998) muito contribuiria para nortear as pesquisas em desenvolvimento humano.

O presente estudo traz significativas contribuições para a compreensão de como se desenvolvem as interações parentais em famílias de crianças com síndrome de Down. As atividades realizadas nas famílias SD exigiam das crianças menos habilidades cognitivas, uma vez que se observou uma baixa freqüência de atividades como 'Jogos' e 'Brincadeiras de atividades escolares', e freqüências mais elevadas em atividades como brincadeiras 'Com objetos', de 'Manipular o corpo' e 'Estouvadas', consideradas mais simples. Por outro lado, o fato de a 'Brincadeira de atividades escolares' ter ocorrido com mais freqüência nas famílias DT, sugere que as famílias SD percebem e se engajam em atividades apropriadas ao nível de desenvolvimento da criança. Merece destaque, também, a atividade de 'Contar histórias', mais freqüente nas famílias SD que nas DT, que pode estar naturalmente contribuindo para o desenvolvimento de habilidades de compreensão e linguagem e favorecendo a atenção e concentração da criança.

O modo como os genitores mudam de uma atividade para outra sugere que eles estão exercendo o seu papel de pais de forma apropriada ao estágio de desenvolvimento de suas crianças, indicando um equilíbrio nas relações parentais, conforme proposto pela abordagem sistêmica da família (Minuchin, 1988). Quando as crianças são pequenas, a responsabilidade maior pelo controle e liderança nas interações é dos genitores e não das crianças. Esses dados são corroborados por Dessen (1992), em se tratando de transições entre genitores e suas crianças com desenvolvimento típico, o que reafirma a não existência de diferenças nos modos como as famílias de crianças com desenvolvimento típico mudam de uma atividade para outra, em comparação com as famílias de crianças com síndrome de Down.
Com relação aos padrões de qualidade, os episódios interativos caracterizaram-se predominantemente pela 'Sincronia' entre genitores-criança, o que significa que os comportamentos emitidos, em sua maioria, tiveram uma resposta adequada do receptor, seja a criança ou os genitores. Portanto, o processo de comunicação entre os membros de ambos os tipos de família pode ser considerado adequado no que tange à dimensão 'Sincronia', particularmente em se tratando de famílias de crianças SD (Colnago, 1991; Pereira-Silva \& Dessen, 2001, 2003; Sigolo, 2004). Por outro lado, não se pode deixar de ressaltar que as famílias SD tiveram um percentual mais elevado de interações parentais 'Sem sincronia', sugerindo que a imaturidade social e cognitiva da criança SD pode estimular respostas inadequadas e não contingentes, embora não suficientes para inviabilizar o processo de comunicação entre genitores e criança SD.

A 'Liderança' nas interações da família DT foi exercida pela mãe e pela criança em proporções similares, sendo o pai responsável por uma freqüência menor de 'Liderança'. Nas famílias SD, o pai e a mãe exerceram a 'Liderança' de forma similar, sugerindo que pais de crianças SD são mais participativos que pais de crianças DT, pelo menos quando interagindo com a criança em atividades livres. No entanto, esses dados são contraditórios com aqueles da literatura, cujos pais são tidos como menos participativos e menos engajados em interações com sua criança DM (Floyd et al., 1997; Floyd \& Zmich, 1991; Sigman, Mundy, Sherman \& Ungerer, 1986). Evidentemente, a participação do pai precisa ser melhor investigada no contexto das famílias brasileiras (Dessen \& Lewis, 1998, Lewis \& Dessen, 1999), tanto de crianças DT quanto SD.

Ainda considerando a dimensão de 'Liderança' nas interações parentais, chamamos a atenção para o fato de que as mães de crianças SD e DT lideraram as interações de forma similar. Este dado também não corrobora os da literatura que apontam uma maior diretividade nas interações de mães com suas crianças DM que de mães com suas crianças DT (Cielinski et al., 1995; Landry et al., 1994; Lieberman et al., 1995; Marfo et al., 1998; Sigolo, 1994, 2000). Estudos futuros deveriam dedicar-se a este tópico, uma vez que esta similaridade entre os grupos de mães pode indicar que os fatores culturais exercem um forte impacto no curso das interações familiares, no contexto brasileiro. No entanto, não podemos descartar a possibilidade de diferenças conceituais em relação aos termos liderança e diretividade.

No decorrer de dois anos, verificamos que alguns padrões de interação permaneceram estáveis, enquanto outros decresceram ou aumentaram. Em ambos os tipos de famílias, as alterações mais acentuadas foram registradas na $2^{\text {a }}$ fase de coleta de dados, particularmente nas interações parentais. Acreditamos que tais alterações se devem, em parte, ao fato de a família sentir-se mais relaxada com a presença da pesquisadora no segundo momento de coleta, havendo um posterior equilíbrio nas fases subseqüentes.

Com relação à qualidade das interações parentais, observamos que enquanto nas famílias DT a 'Sincronia' e 
'Amistosidade' mostram uma ligeira tendência a aumentar, o que é compatível com o progresso no desenvolvimento cognitivo e social da criança, nas famílias SD o mesmo não ocorre. Nestas famílias, estas dimensões permaneceram em um patamar estável e baixo, o que demanda procedimentos de intervenção dada à importância da qualidade das interações para promoção do desenvolvimento infantil (Dessen \& Pereira-Silva, 2004; Sigolo, 2004). Pais e mães devem ser estimulados a desenvolver interações mais sincrônicas e amistosas com suas crianças SD. Em contrapartida, as interações 'Sem sincronia' e 'Conflituosas' entre genitores e sua criança tendem a diminuir ao longo do tempo, em ambos os tipos de família. Acreditamos que esta tendência reflete, novamente, uma sintonia dos genitores ao lidar com suas crianças, coerente com o estágio de desenvolvimento em que elas se encontram.

Os padrões de interações parentais que emergiram das análises deste estudo merecem investigações futuras a partir de uma amostra significativa de famílias SD e DT, que considere outros contextos ecológicos, como o ambiente da escola, de extrema importância para a socialização da criança com deficiência. Os nossos dados sugerem, também, a necessidade de os programas de intervenção e prevenção serem direcionados às famílias e não especificamente à criança com deficiência, priorizando as interações entre os diferentes subsistemas, conforme proposto, recentemente, por Dessen e Pereira-Silva (2004).

\section{Referências}

Berger, J. (1990). Interactions between parents and their infants with Down syndrome. In D. Cicchetti \& M. Beeghly (Eds.), Children with Down syndrome: A developmental perspective (pp. 101-146). Cambridge, UK: Cambridge University Press.

Bronfenbrenner, U. (1999). Environments in developmental perspective: Theoretical and operational models. In S. L. Friedman \& T. D. Wachs (Eds.), Measuring environment across the life span: Emerging methods and concepts (pp. 3-28). Washington, DC: American Psychological Association.

Bronfenbrenner, U., \& Morris, P. A. (1998). The ecology of developmental processes. In W. Damon \& R. M. Lerner (Eds.), Handbook of child psychology: Theoretical models of human development (Vol. 1, pp. 993-1028). New York: Wiley.

Cielinski, K. L., Vaughn, B. E., Seifer, R., \& Contreras, J. (1995). Relations among sustained engagement during play, quality of play, and mother-child interaction in samples of children with Down syndrome and normally developing toddlers. Infant Behavior and Development, 18, 163-176.

Colnago, N. A. S. (1991). Pares "mães-bebês síndrome de Down": Estudo da estimulação e dos aspectos qualitativos da interação. Dissertação de Mestrado não-publicada, Universidade Federal de São Carlos, SP.

Dessen, M. A. (1992). Efeitos do nascimento de uma segunda criança no comportamento e nas relações entre o primogênito e os genitores. Tese de Doutorado não-publicada, Universidade de São Paulo, SP.
Dessen, M. A. (1997). Desenvolvimento familiar: Transição de um sistema triádico para poliádico. Temas em Psicologia, 3, 51-61.

Dessen, M. A., \& Lewis, C. (1998). Como estudar a família e o «pai»? Cadernos de Psicologia e Educação Paidéia, 8, 105-121.

Dessen, M. A., \& Pereira-Silva, N. L. (2000). Deficiência mental e família: Uma análise da produção científica. Cadernos de Psicologia e Educação Paidéia, 10, 12-23.

Dessen, M. A., \& Pereira-Silva, N. L. (2004). A família e os programas de intervenção: Tendências atuais. In E. G. Mendes, M. A. Almeida \& L. C. A.Williams, Temas em Educação Especial: Avanços recentes (pp. 179-188). São Carlos, SP: EdUFSCar.

Floyd, F. J., Costigan, C. L., \& Phillippe, K. A. (1997). Developmental change and consistency in parental interactions with school-age children who have mental retardation. American Journal on Mental Retardation, 101, 579-594.

Floyd, F. J., \& Zmich, D. E. (1991). Marriage and the parenting partnership: Perceptions and interactions of parents with mentally retarded and typically developing children. Child Development, 62, 1434-1448.

Hornby, G. (1995). Effects on fathers of children with Down syndrome. Journal of Child and Family Studies, 4, 239-255.

Kreppner, K. (2000). The child and the family: Interdependence in developmental pathways. Psicologia: Teoria e Pesquisa, 16, 11-22.

Lamb, M .E., \& Billings, L. A. L. (1997). Fathers of children with special needs. In M. E. Lamb (Ed.), The role of the father in child development (pp. 179-190). New York: Wiley.

Landry, S. H., Garner, P. W., Pirie, D., \& Swank, P. R. (1994). Effects of social context and mothers' requesting strategies on Down's syndrome children's social responsiveness. Developmental Psychology, 30, 293-302.

Lewis, C., \& Dessen, M. A. (1999). O pai no contexto familiar. Psicologia: Teoria e Pesquisa, 15, 09-16.

Lieberman, D., Padan-Belkin, E., \& Harel, S. (1995). Maternal directiveness and infant compliance at one year of age: $\mathrm{A}$ comparison between mothers and their developmentallydelayed infants and mothers and their nondelayed infants. Journal of Child Psychology and Psychiatry and Allied Disciplines, 36, 1091-1096.

Marfo, K., Dedrick, C. F., \& Barbour, N. (1998). Mother-child interactions and development of children with mental retardation. In J. A. Burack, R. M. Hodapp \& E. Zigler (Eds.), Handbook of mental retardation and development (pp. 637-668). Cambridge, UK: Cambridge University Press.

Minuchin, P. (1988). Relationships within the family: A systems perspective on development. In R. A. Hinde \& J. StevensonHinde (Eds.), Relationships within families: Mutual influences. Oxford, UK: Clarendon Press.

Pereira-Silva, N. L. (2003). Famílias de crianças com e sem síndrome de Down: Um estudo comparativo das relações familiares. Tese de Doutorado não-publicada, Universidade de Brasília, DF.

Pereira-Silva, N. L., \& Dessen, M. A. (2001). Deficiência mental e família: Implicações para o desenvolvimento da criança. Psicologia: Teoria e Pesquisa, 17, 133-141.

Pereira-Silva, N. L., \& Dessen, M. A. (2003). Crianças com síndrome de Down e suas interações familiares. Psicologia: Reflexão e Crítica, 16, 503-514.

Roach, M. A., Barrat, M. S., Miller, J. F., \& Leavitt, L. A. (1998). The structure of mother-child play: Young children with Down syndrome and typically developing children. Developmental Psychology, 34, 77-87. 
Rodrigue, J. R., Morgan, S. B., \& Geffken, G. R. (1992). Psychosocial adaptation of fathers of children with autism, Down syndrome and normal development. Journal of Autism and Development Disorders, 22, 249-263.

Sigman, M., Mundy, P., Sherman, T., \& Ungerer, J. (1986). Social interactions of autistic, mentally retarded and normal children and their caregivers. Journal of Child Psychology and Psychiatry and Allied Disciplines, 27, 647-656.

Sigolo, S. R. R. L. (1994). Análise da interação mãe-criança com atraso de desenvolvimento no segundo ano de vida. Tese de Doutorado não-publicada, Universidade de São Paulo, SP.

Sigolo, S. R. R. L. (2000). Diretividade materna e socialização de crianças com atraso de desenvolvimento. Cadernos de Psicologia e Educação Paidéia, 10, 47-54.

Sigolo, S. R. R. L. (2004). Favorecendo o desenvolvimento infantil: Enfase nas trocas interativas no contexto familiar. In E. G. Mendes, M. A. Almeida \& L. C. A. Williams, Temas em Educação Especial: Avanços recentes (pp. 189-195). São Carlos, SP: Editora da Universidade Federal de São Carlos.

Vaughn, B. E., Goldberg, S., Atkinson, L., Marcovitch, S., MacGregor, D., \& Seifer, R. (1994). Quality of toddler-mother attachment in children with Down syndrome: Limits to interpretation of strange situation behavior. Child Development, 65, 95-108. 